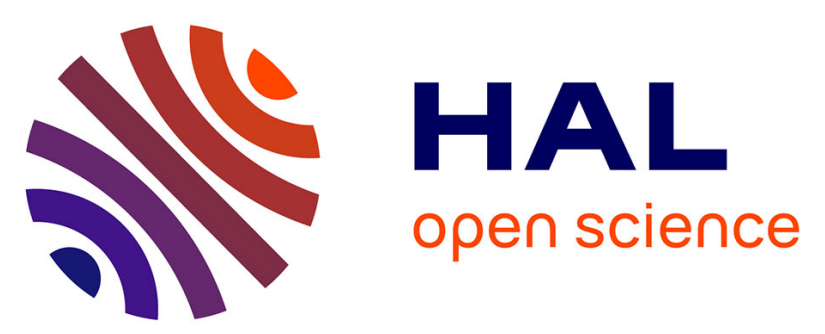

\title{
Theoretical and numerical investigations of frequency analysis of two circular cylinders oscillating in a incompressible viscous fluid
}

Adil El Baroudi, Fulgence Razafimahéry

\section{- To cite this version:}

Adil El Baroudi, Fulgence Razafimahéry. Theoretical and numerical investigations of frequency analysis of two circular cylinders oscillating in a incompressible viscous fluid. International Journal of Applied Mechanics, 2014, 6 (5), pp.1-20. 10.1142/S1758825114500495 . hal-01207439

HAL Id: hal-01207439

https://hal.science/hal-01207439

Submitted on 30 Sep 2015

HAL is a multi-disciplinary open access archive for the deposit and dissemination of scientific research documents, whether they are published or not. The documents may come from teaching and research institutions in France or abroad, or from public or private research centers.
L'archive ouverte pluridisciplinaire HAL, est destinée au dépôt et à la diffusion de documents scientifiques de niveau recherche, publiés ou non, émanant des établissements d'enseignement et de recherche français ou étrangers, des laboratoires publics ou privés. 


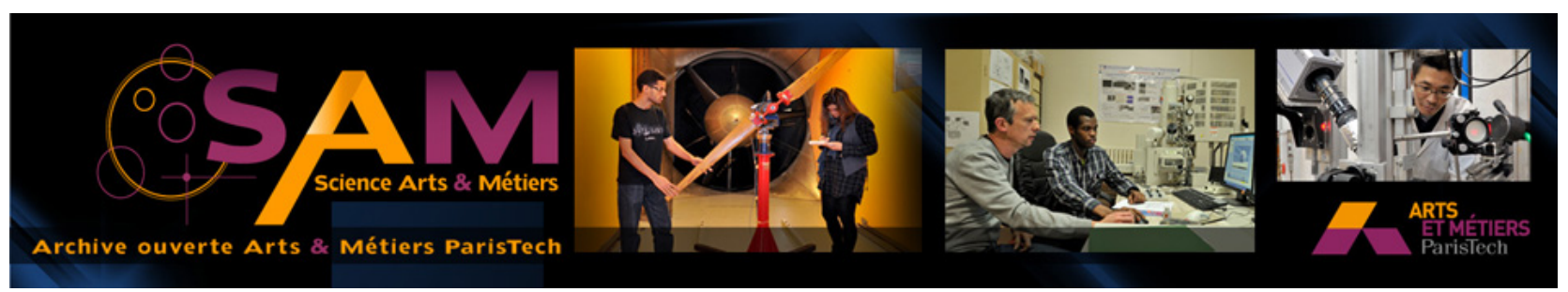

\section{Science Arts \& Métiers (SAM)}

is an open access repository that collects the work of Arts et Métiers ParisTech researchers and makes it freely available over the web where possible.

This is an author-deposited version published in: http://sam.ensam.eu

Handle ID: .http://hdl.handle.net/10985/10284

\section{To cite this version :}

Adil EL BAROUDI, Fulgence RAZAFIMAHERY - Theoretical and numerical investigations of frequency analysis of two circular cylinders oscillating in a incompressible viscous fluid International Journal of Applied Mechanics - Vol. 6, n5, p.1-20 - 2014 


\title{
THEORETICAL AND NUMERICAL INVESTIGATIONS OF FREQUENCY ANALYSIS OF TWO CIRCULAR CYLINDERS OSCILLATING IN A INCOMPRESSIBLE VISCOUS FLUID
}

\author{
ADIL EL BAROUDI \\ Arts et Métiers ParisTech, 2 Boulevard du Ronceray \\ 49035 Angers, France \\ adil.elbaroudi@ensam.eu \\ FULGENCE RAZAFIMAHERY \\ IRMAR \\ Université de Rennes 1, Campus de Beaulieu \\ 35042 Rennes Cedex, France \\ fulgence.razafimahery@univ-rennes1.fr
}

\begin{abstract}
A potential flow is presented in this paper for the analysis of the fluid-structure interaction systems including, but not limited to, the idealized human head. The model considers a cerebro-spinal fluid (CSF) medium interacting with two solid domain. The fluid field is governed by the linearized Navier-Stokes equation. A potential technique is used to obtain a general solution for a problem. The method consists in solving analytically partial differential equations obtained from the linearized Navier-Stokes equation. From the solution, modal shapes and stokes cells are shown. In the analysis, the elastic skull model and the rigid skull model are presented. A finite element analysis is also used to check the validity of the present method. The results from the proposed method are in good agreement with numerical solutions. The effects of the fluid thickness is also investigated.
\end{abstract}

Keywords: Fluid-structure interactions; coupled vibration; finite element method; Helmholtz decomposition; modal analysis.

\section{Introduction}

Fluid-structure interaction problems since long have attracted the attention of engineers and applied mathematics. The most important applications of this theory, probably, structural acoustics [Ohayon and Soize, 1997], vibrations of fluidconveying pipes [Paidoussis, 1997] and biomechanics. As these problems are rather complicated, some simplifications are typically adopted to facilitate their solving. In particular, it is quite typical to ignore viscosity effects (especially in structural 
acoustics) or to use local theories of interaction, such as, the one referred to as thin layer or plane wave approximation.

In recent years, several authors have focused on the modeling of inertial coupling of the brain, cerebro-spinal fluid (CSF) and skull system. This model of inertial coupling was solved using FEM method [Chen and Luo, 2010] and analytical method [El Baroudi et al., 2012a]. The reported study in El Baroudi et al. [2009], the viscosity effects in fluid-structure interaction problem studied are neglected. Indeed, the perfect fluid model is used to describe the interactions between fluid and solids. The fluid is assumed nonviscous and isotropic which satisfies the acoustic wave equation. Since no source is considered in the acoustic domain, the Helmholtz equation, which describes a harmonic wave equation propagating in medium while neglecting dissipation, is represented as $\nabla^{2} p+\omega^{2} / c^{2} p=0$ where $p$ is the acoustic pressure in a fluid medium, $\omega$ is the angular frequency and speed of sound $c$. In this case, the coupling is considered to be inertial. In fact, in linear systems fluid inertia can be modeled as an added mass matrix operating on the degrees of freedom of the structure, which means that no additional degree of freedom related to the fluid is required. When the CSF's thickness becomes very low, is required to take into account of character of viscous flow [Axisa and Antunes, 2007]. On one hand, brain injuries constitute one of the major cause of death in road accidents. To understand how the brain gets injured during an accident, the mechanical response of the contents of the head during an impact has to be known. Several models have been developed to study this phenomenon. Some authors used numerical models using the finite element method [Marjoux et al., 2008; Liu et al., 2007], while others developed experimental devices that simulate a shock [Verschuerena et al., 2007]. Most numerical models used did not always show the coupled character of the problem studied [Zong et al., 2006]. The consequences of the skull's vibrations are still poorly understood. It is probable that the low-frequency skull vibrations (below $200 \mathrm{~Hz}$ ) mainly cause deep cerebral lesions, while higher frequency vibrations have more consequences on the superficial cerebral structures [Willinger et al., 1995].

On the other hand, the knowledge of the flow dynamics and oscillations of the $\mathrm{CSF}$ in the intra-cranial space plays an important role for various human pathology in the everyday activities. More recent studies on the brain tend more and more to point out the role of wave within the brain tissue e.g., Will and Berg [2007]. They experimentally showed that synchronization responses of brainwaves to periodic auditory stimuli had three components corresponding to different spectra: low frequencies $[1-5 \mathrm{~Hz}]$, intermediate frequencies $[3-8 \mathrm{~Hz}]$ and higher frequencies [11-44 Hz]. In addition, mechanical vibrations also play important role during measurement as Diffusion Tensor Imaging by means of Magnetic Resonance Imaging. Recent studies showed that the mechanical vibrations cannot be ignored and they should be considered when choosing the sequence parameters for Diffusion Tensor Imaging [Hiltunen et al., 2006]. All these observations suggest us to better determine the natural frequencies and modal shapes of the brain, the CSF and the skull. 
Analytical methods were conducted to determine the frequency spectrum of the head-neck system [Charalambopoulos et al., 2006]. Finite element method has been intensively used to model the head impact [Kleiven, 2002]. Most of them neglected the mechanical role of the CSF. A basic question remain: what would be the role of the CSF for free and forced vibrations of the brain-CSF-skull system at low frequencies $\leq 200[\mathrm{~Hz}]$ ? Numerical aspects of fluid-structure problems have been studied in the past [Morand et al., 1979]. Most classical methods use the modal shapes of the solid phase for deriving the dynamical behavior of the whole (solid and fluid domains). In a general manner, classical methods may be sufficient if we are only interested in searching for the natural frequencies. However, these methods do not permit to obtain the modal shapes of the whole system which is essential for applying the modal projection when facing dynamical situation (forced vibrations or shocks).

The aim of the present work was to search for analytical and numerical modal shapes of the interacting skull-CSF-brain assuming elastic behavior for the brain and the skull and an acoustic wave propagation within the CSF. The starting point for our study is based on the experimental devices which may be used in biomechanical modeling of the human head. Most of these experimental devices which consist of two concentric cylinders separated by a liquid media (water), the water layer thickness used is much more higher than the actual value of CSF thickness. This led us to use the inertial coupling model proposed in the study [El Baroudi et al., 2009]. In this paper, we propose a model based on viscous coupling between two circular cylinders separated by a viscous fluid, which is better suited to the small CSF thickness. In the reported study in El Baroudi et al. [2012b], we also carried out an analytical and numerical study for a spherical head in the case of an inertial coupling. The model (Fig. 1) is designed to directly solve the viscous coupled solid-fluid problem, conversely to classical method that only uses the modal shapes of the solid phase. The influence of the CSF compressibility, dynamic viscosity and thickness on the natural frequencies and modal shapes was investigated. Last but not least, the use of simple models was deemed necessary since validation of the fluid-structure problems still remains a challenge.

\section{Mathematical Formulation of the Problem}

\subsection{Governing differential equations of viscous fluid region $\Omega_{f}$}

From conservations of mass and momentum and assuming that the fluid is viscous and incompressible, the motion of the fluid flowing in the cylindrical waveguide in the absence of body force is governed by

$$
\begin{gathered}
\rho \frac{\partial \mathbf{v}}{\partial t}=\nabla \cdot\left\{-p \mathbf{I}+\eta\left[\nabla \mathbf{v}+(\nabla \mathbf{v})^{T}\right]\right\} \\
\nabla \cdot \mathbf{v}=0,
\end{gathered}
$$




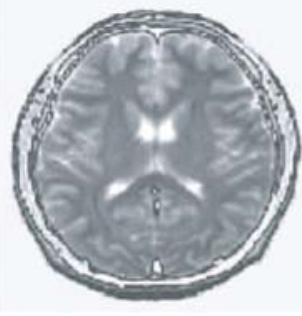

(a) Brain system

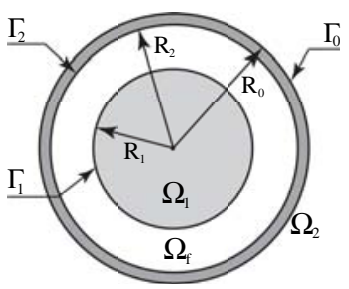

(c) Elastic skull

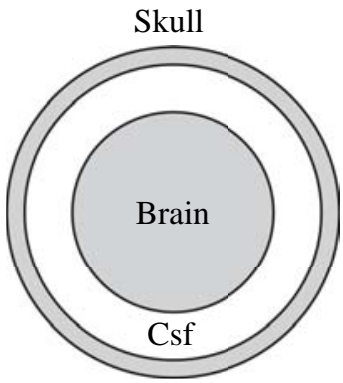

(b) Geometry of domain

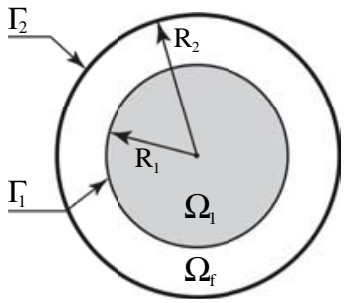

(d) Rigid skull

Fig. 1. Top view of the Brain (a): 2D Geometry of domain, (b): Elastic model, (c): brain $\left(\Omega_{1}\right)$, $\operatorname{csf}\left(\Omega_{f}\right)$ and skull $\left(\Omega_{2}\right)$, Rigid model and $(\mathrm{d})$ : brain $\left(\Omega_{1}\right)$ and $\operatorname{csf}\left(\Omega_{f}\right)$.

in which $\mathbf{v}=\left\{v_{r}, v_{\theta}\right\}^{\mathrm{T}}$ is the fluid velocity vector, $p$ is the fluid pressure, $\rho$ is the density of the fluid, $\nu$ and $\eta=\rho \nu$ are the kinematic and dynamic fluid viscosity, respectively. $\mathbf{I}$ is a unit tensor.

The obtained equations of motion are highly complex and coupled. However, a simpler set of equations can be obtained by introducing scalar potentials $\phi$ and $\psi$, known as the Helmholtz decomposition. The Helmholtz decomposition theorem [Morse and Feshbach, 1946] states that any vector $\mathbf{v}$ can be written as the sum of two parts, one is curl-free and the other is solenoidal. In flow fields, the velocity is thereby decomposed into a potential flow and a viscous flow. In other words, the velocity $\mathbf{v}$ can be expressed as a sum of the gradient of a scalar potential $\phi$ and the curl of a vector potential $\boldsymbol{\Psi}$ as follows:

$$
\mathbf{v}=\nabla \phi+\nabla \times \mathbf{\Psi}
$$

where $\boldsymbol{\Psi}$ is a vector stream function. Using the problem symmetry, the vector potential $\boldsymbol{\Psi}$ reduces to a scalar equation; i.e., $\boldsymbol{\Psi}=(0,0, \psi)$, using the condition $\nabla \cdot \boldsymbol{\Psi}=0$, and substituting the above resolutions into Eqs. (1) and (2), after some manipulations, the equation for the conservation of mass (2) becomes the Laplace equation 
and the equation for the conservation of momentum (1) becomes

$$
\begin{aligned}
\nabla^{2} \psi-\frac{1}{\nu} \frac{\partial \psi}{\partial t} & =0 \\
p+\rho \frac{\partial \phi}{\partial t} & =0
\end{aligned}
$$

where $\nabla^{2}=\frac{\partial^{2}}{\partial r^{2}}+\frac{1}{r} \frac{\partial}{\partial r}+\frac{1}{r^{2}} \frac{\partial^{2}}{\partial \theta^{2}}$ is the Laplacian operator in polar coordinates $(r, \theta)$. Thus, the Navier-Stokes equations are reduced to formulation (4) and (5). Of course, it does not mean that in any case such a decomposition of fluid's velocity field gives considerable simplifications in solving of the problem because boundary conditions for stresses are not separated. The vibrations are harmonic, with constant angular frequency $\omega$, as a result all potentials components and pressure will depend on time only through the factor $\exp (j \omega t)$. Applying the method of separation of variables, the solution of the equations for potentials, associated with an axial wave number $k_{z}$, radial wave number $k_{\psi}$ and circumferential mode parameter $n$, after considerable algebraic manipulations, can be shown to be

$$
\begin{gathered}
\phi=\left[A \phi_{1}(r)+B \phi_{2}(r)\right]\left\{\begin{array}{l}
\sin (n \theta) \\
\cos (n \theta)
\end{array}\right\}, \\
\phi_{1}(r)=r^{n}, \phi_{2}(r)=r^{-n}, \\
\psi=\left[C \psi_{1}(r)+D \psi_{2}(r)\right]\left\{\begin{array}{l}
\cos (n \theta) \\
\sin (n \theta)
\end{array}\right\}, \\
\psi_{1}(r)=J_{n}\left(k_{\psi} r\right), \quad \psi_{2}(r)=Y_{n}\left(k_{\psi} r\right) .
\end{gathered}
$$

$J_{n}$ and $Y_{n}$ are Bessel functions of the first and second kind of order $n . n=0,1,2, \ldots$ is the azimuthal wavenumber. Note that the velocity field $\mathbf{v}$ has components that are symmetric or antisymmetric in $\theta$. The azimuthal modes corresponding to $\cos (n \theta)$ and $\sin (n \theta)$ are really the same, due to periodicity in the azimuthal direction, i.e., there is no distinction in the values of $n$ for the two families. $A, B, C, D, E$ and $F$ are unknown coefficients which will be determined later by imposing the appropriate boundary conditions. The radial wave number $k_{\psi}$ is related to the angular frequency $\omega$ by

$$
k_{\psi}^{2}=-\frac{j \omega}{\nu}, \quad j=\sqrt{-1}
$$

Using Eq. (3) and taking into account Eq. (6), the fluid particle velocities in terms of the Bessel functions can be written as

$$
\begin{aligned}
& v_{r}=\left\{A \frac{d \phi_{1}}{d r}(r)+B \frac{d \phi_{2}}{d r}(r)-\frac{n}{r} C \psi_{1}(r)-\frac{n}{r} D \psi_{2}(r)\right\} \sin (n \theta), \\
& v_{\theta}=\left\{\frac{n}{r} A \phi_{1}(r)+\frac{n}{r} B \phi_{2}(r)-C \frac{d \psi_{1}}{d r}(r)-D \frac{d \psi_{2}}{d r}(r)\right\} \cos (n \theta) .
\end{aligned}
$$


The radial and tangential stresses for an incompressible viscous fluid (CSF) in terms of the potentials $\phi$ and $\psi$ can be written as

$$
\begin{aligned}
\sigma_{r r}= & \eta\left\{2 \frac{d^{2} \phi_{1}}{d r^{2}}(r)-k_{\psi}^{2} \phi_{1}(r)\right] A+\left[2 \frac{d^{2} \phi_{2}}{d r^{2}}(r)-k_{\psi}^{2} \phi_{2}(r)\right] B \\
& \left.-\frac{2 n}{r^{2}}\left[r \frac{d \psi_{1}}{d r}(r)-\psi_{1}(r)\right] C-\frac{2 n}{r^{2}}\left[r \frac{d \psi_{2}}{d r}(r)-\psi_{2}(r)\right] D\right\} \sin (n \theta), \\
\sigma_{r \theta}= & \eta\left\{\frac{2 n}{r^{2}}\left[r \frac{d \phi_{1}}{d r}(r)-\phi_{1}(r)\right] A+\frac{2 n}{r^{2}}\left[r \frac{d \phi_{2}}{d r}(r)-\phi_{2}(r)\right] B\right. \\
& \left.-\left[2 \frac{d^{2} \psi_{1}}{d r^{2}}(r)+k_{\psi}^{2} \psi_{1}(r)\right] C-\left[2 \frac{d^{2} \psi_{2}}{d r^{2}}(r)+k_{\psi}^{2} \psi_{2}(r)\right] D\right\} \cos (n \theta) .
\end{aligned}
$$

\subsection{Governing differential equations of elastic solids region $\Omega_{i}$}

The cylinders are assumed to be made of an elastic solids with Young's modulus $E_{i}$, Poisson ratio $\nu_{i}$, mass density $\rho_{i}$ and $i=1,2$ indicates the brain $(i=1)$ and the skull $(i=2)$, respectively. For motion in a homogeneous isotropic elastic solid, the vector displacement $\mathbf{u}^{(i)}(r, \theta)=\left\{u_{r}^{(i)}, u_{\theta}^{(i)}\right\}^{\mathrm{T}}$ satisfies Navier's equation. Using Helmholtz decomposition method, the displacement vector within the body of an elastic solid can be written in terms of two scalar and vector potential functions

$$
\mathbf{u}^{(i)}=\nabla \Phi^{(i)}+\nabla \times \Psi^{(i)}, \quad \Psi^{(i)}=\left(0,0, \Psi^{(i)}\right)^{T} .
$$

By expanding Eq. (10) and substituting it into Navier's equation

$$
-\rho_{i} \omega^{2} \mathbf{u}^{(i)}=\mu_{i} \nabla^{2} \mathbf{u}^{(i)}+\left(\lambda_{i}+\mu_{i}\right) \nabla \nabla \cdot \mathbf{u}^{(i)},
$$

where $\rho_{i}$ is the density, $\lambda_{i}, \mu_{i}$ are the Lamé constants, $r$ is the radial direction and $\theta$ is the circumferential direction. Two partial differential equations are obtained in terms of the three potential functions

$$
\nabla^{2} \Phi^{(i)}-\frac{\nu^{2} k_{\psi}^{4}}{c_{L_{i}}^{2}} \Phi^{(i)}=0, \quad \nabla^{2} \Psi^{(i)}-\frac{\nu^{2} k_{\psi}^{4}}{c_{T_{i}}^{2}} \Psi^{(i)}=0,
$$

where $c_{L_{i}}=\sqrt{\frac{\lambda_{i}+2 \mu_{i}}{\rho_{i}}}$ and $c_{T_{i}}=\sqrt{\frac{\mu_{i}}{\rho_{i}}}$ are the compressional and shear wave velocities in the solids, respectively. Similarly, the solution of the equations for potentials, associated with a longitudinal and shear wave numbers $\left(k_{L_{i}}, k_{T_{i}}\right)$ in the solid media and circumferential mode parameter $n$, can be shown to be

$$
\begin{aligned}
& \Phi^{(i)}=\left[A_{i} \Phi_{1}^{(i)}(r)+B_{i} \Phi_{2}^{(i)}(r)\right] \sin (n \theta), \\
& \Psi^{(i)}=\left[C_{i} \Psi_{1}^{(i)}(r)+D_{i} \Psi_{2}^{(i)}(r)\right] \cos (n \theta),
\end{aligned}
$$


where

$$
\begin{aligned}
& \Phi_{1}^{(i)}(r)=I_{n}\left(k_{L_{i}} r\right), \quad \Phi_{2}^{(i)}(r)=K_{n}\left(k_{L_{i}} r\right), \quad k_{L_{i}}=\frac{\nu}{c_{L_{i}}} k_{\psi}^{2}, \\
& \Psi_{1}^{(i)}(r)=I_{n}\left(k_{T_{i}} r\right), \quad \Psi_{2}^{(i)}(r)=K_{n}\left(k_{T_{i}} r\right), \quad k_{T_{i}}=\frac{\nu}{c_{T_{i}}} k_{\psi}^{2},
\end{aligned}
$$

and the unknown coefficients $A_{i}, B_{i}, C_{i}$ and $D_{i}$ are to be determined from boundary conditions. $I_{n}$ and $K_{n}$ are modified Bessel functions of the first and second kind of order $n$. Also, using Eq. (10) and Hooke's law, the scalar components of the displacement vector $\mathbf{u}^{(i)}$ and the stress tensor $\Sigma^{(i)}$ of the elastic medium in terms of the potentials $\Phi_{i}$ and $\Psi_{i}$ can be written as

$$
\begin{aligned}
u_{r}^{(i)}= & \left\{A_{i} \frac{d \Phi_{1}^{(i)}}{d r}(r)+B_{i} \frac{d \Phi_{2}^{(i)}}{d r}(r)-\frac{n}{r} C_{i} \Psi_{1}^{(i)}(r)-\frac{n}{r} D_{i} \Psi_{2}^{(i)}(r)\right\} \sin (n \theta), \\
u_{\theta}^{(i)}= & \left\{\frac{n}{r} A_{i} \Phi_{1}^{(i)}(r)+\frac{n}{r} B_{i} \Phi_{2}^{(i)}(r)-C_{i} \frac{d \Psi_{1}^{(i)}}{d r}(r)-D_{i} \frac{d \Psi_{2}^{(i)}}{d r}(r)\right\} \cos (n \theta), \\
\Sigma_{r r}^{(i)}= & 2 \mu_{i}\left\{A_{i}\left[\frac{d^{2} \Phi_{1}^{(i)}}{d r^{2}}(r)+\frac{\lambda_{i} k_{L_{i}}^{2}}{2 \mu_{i}} \Phi_{1}^{(i)}(r)\right]+B_{i}\left[\frac{d^{2} \Phi_{2}^{(i)}}{d r^{2}}(r)+\frac{\lambda_{i} k_{L_{i}}^{2}}{2 \mu_{i}} \Phi_{2}^{(i)}(r)\right]\right. \\
& \left.-C_{i} \frac{n}{r^{2}}\left[r \frac{d \Psi_{1}^{(i)}}{d r}(r)-\Psi_{1}^{(i)}(r)\right]-D_{i} \frac{n}{r^{2}}\left[r \frac{d \Psi_{2}^{(i)}}{d r}(r)-\Psi_{2}^{(i)}(r)\right]\right\} \sin (n \theta), \\
\Sigma_{r \theta}^{(i)}= & 2 \mu_{i}\left\{A_{i} \frac{n}{r^{2}}\left[r \frac{d \Phi_{1}^{(i)}}{d r}(r)-\Phi_{1}^{(i)}(r)\right]+B_{i} \frac{n}{r^{2}}\left[r \frac{d \Phi_{2}^{(i)}}{d r}(r)-\Phi_{2}^{(i)}(r)\right]\right. \\
& \left.-C_{i}\left[\frac{d^{2} \Psi_{1}^{(i)}}{d r^{2}}(r)-\frac{k_{T_{i}}^{2}}{2} \Psi_{1}^{(i)}(r)\right]-D_{i}\left[\frac{d^{2} \Psi_{2}^{(i)}}{d r^{2}}(r)-\frac{k_{T_{i}}^{2}}{2} \Psi_{2}^{(i)}(r)\right]\right\} \cos (n \theta),
\end{aligned}
$$

\section{Fluid-Structure Interaction and Frequency Equation}

First we define $\Gamma_{1}:\left(r=R_{1}\right)$ as the boundary contact between the fluid (CSF) region $\left(\Omega_{f}\right)$ and the brain region $\left(\Omega_{1}\right)$, and $\Gamma_{2}:\left(r=R_{2}\right)$ as the boundary contact between the fluid (CSF) region $\left(\Omega_{f}\right)$ and the skull region $\left(\Omega_{2}\right)$, and $\Gamma_{0}:\left(r=R_{0}\right)$ as the outer boundary (the outer interface of the skull).

\subsection{Frequency equation in the deformable skull case}

The relevant boundary conditions those are physically realistic and mathematically consistent for this problem at the inner and outer surfaces of the elastic solid (brain) 
in contact with fluid medium (CSF) can be taken as follows:

- On the interface $\Gamma_{1}$, the displacements (velocities), normal and the tangential stresses must be continuous, leading to:

$$
\begin{gathered}
v_{r}=j \omega u_{r}^{(1)}, \quad v_{\theta}=j \omega u_{\theta}^{(1)}, \\
\sigma_{r r}=\Sigma_{r r}^{(1)}, \quad \sigma_{r \theta}=\Sigma_{r \theta}^{(1)} .
\end{gathered}
$$

- On the interface $\Gamma_{2}$, the displacements (velocities), normal and the tangential stresses must be continuous, leading to:

$$
\begin{gathered}
v_{r}=j \omega u_{r}^{(2)}, \quad v_{\theta}=j \omega u_{\theta}^{(2)}, \\
\sigma_{r r}=\Sigma_{r r}^{(2)}, \quad \sigma_{r \theta}=\Sigma_{r \theta}^{(2)} .
\end{gathered}
$$

- On the interface $\Gamma_{0}$ the normal and the tangential stresses must be zero, leading to:

$$
\Sigma_{r r}^{(2)}=\Sigma_{r \theta}^{(2)}=0
$$

Combining these boundary conditions with Eqs. (8), (9), (15) and (16) yields for each mode number $n$ the following linear system

$$
[\mathbf{M}]\{\mathbf{x}\}=\{\mathbf{0}\}, \quad\{\mathbf{x}\}=\left\{\begin{array}{llllllllll}
A_{1} & C_{1} & A & B & C & D & A_{2} & B_{2} & C_{2} & D_{2}
\end{array}\right\}^{T},
$$

where and $[\mathbf{M}]$ is a $10 \times 10$ matrix. For nontrivial solution, the determinant of the matrix $\mathbf{M}$ must be equal to zero

$$
\operatorname{det}[\mathbf{M}]=0 .
$$

This equation indicates a relationship between the dynamic fluid viscosity $\eta$, density of the fluid $\rho$ and the elastic constants. The roots of Eq. (23) give the infinite natural complex frequencies $\omega$.

\subsection{Frequency equation in the case of rigid skull}

In this section, the skull is assumed to be rigid. The problem is governed by Eq. (11) (for $i=1$ ) with boundary conditions Eqs. (17) and (18), and on the interface $\Gamma_{2}$ between the fluid (CSF) and the skull we have

$$
v_{r}=v_{\theta}=0 .
$$

In this case, the matrix $\mathbf{M}$ is reduced to six rows and columns the elements of $\{\mathbf{x}\}$ are reduced to $\left\{\begin{array}{llllll}A_{1} & C_{1} & A & B & C & D\end{array}\right\}^{T}$. 


\subsection{Torsional mode vibration}

The results presented in Eq. (23) are a general natural frequencies equation. For some simpler modes, the above-mentioned method can be simplified. For example: the torsion mode vibration is such a mode in which the scalar components of the displacement $u_{r}^{(i)}$ and the scalar components of the velocity $v_{r}$ are zeros and only the circumferential displacement $u_{\theta}^{(i)}$ and velocity $v_{\theta}$ is independent of $\theta$. This condition is achieved if $\phi=\Phi^{(i)}=0$. Thus, the general solution for $\left\{\psi, \Psi^{(i)}\right\}$ must be constructed from the set

$$
\begin{gathered}
\psi=C J_{0}\left(k_{\psi} r\right)+D Y_{0}\left(k_{\psi} r\right), \\
\Psi^{(i)}=C_{i} I_{0}\left(k_{T_{i}} r\right)+D_{i} K_{0}\left(k_{T_{i}} r\right) .
\end{gathered}
$$

In this case, the boundary conditions equations (17)-(21) becomes

$$
v_{\theta}=j \omega u_{\theta}^{(1)}, \quad \sigma_{r \theta}=\Sigma_{r \theta}^{(1)}, \quad v_{\theta}=j \omega u_{\theta}^{(2)}, \quad \sigma_{r \theta}=\Sigma_{r \theta}^{(2)}, \quad \Sigma_{r \theta}^{(2)}=0 .
$$

Then, Eq. (22) becomes

$$
[\mathbf{T}]\{\mathbf{y}\}=\{\mathbf{0}\}, \quad\{\mathbf{y}\}=\left\{\begin{array}{lllll}
C_{1} & C & D & C_{2} & D_{2}
\end{array}\right\}^{T} .
$$

$[\mathbf{T}]$ is a $5 \times 5$ matrix. Solving $\operatorname{det}[\mathbf{T}]=0$ gives the torsional modes.

\section{Results and Validation}

Numerical calculations were performed on the example of the hollow cylinder (Fig. 1) with different dimensions such as $R_{1}=0.075[\mathrm{~m}], R_{2}=0.09[\mathrm{~m}]$ and $R_{0}=0.095[\mathrm{~m}]$ of the geometry of domain are used. The fluid used (CSF) for which density of $1000\left[\mathrm{~kg} \cdot \mathrm{m}^{-3}\right]$ and dynamic's viscosity of $10^{-3}[\mathrm{~Pa} \cdot \mathrm{s}]$ is assumed. The CSF is comprised of more than $90 \%$ of water, therefore the CSF properties are very close to those of water. The following values of parameters of the elastic solids (brain and skull) is in contact with a viscous liquid were assumed: $\rho_{1}=1150\left[\mathrm{~kg} / \mathrm{m}^{3}\right], \rho_{2}=2750\left[\mathrm{~kg} / \mathrm{m}^{3}\right], \nu_{1}=0.48, \nu_{2}=0.33, E_{1}=6.7 \cdot 10^{5}[\mathrm{~Pa}]$ and $E_{2}=7 \cdot 10^{10}[\mathrm{~Pa}]$.

With the derived eigenfrequency equations, natural frequencies $\omega_{n}=\operatorname{Im}\left(\nu \alpha_{n}^{2} j\right)$ for each $n$ are calculated in the software Mathematica. $n$ denotes the mode in azimuthal (propagating clockwise around the vortex) direction. To validate the analytical results, the natural frequencies and mode shapes are also computed using Comsol Multiphysics [2008] FEM Simulation Software. The natural frequencies are computed directly from Eq. (27) for torsion vibration.

Tables 1 and 2 show the comparison of the first 21 natural frequencies and the corresponding mode shapes of brain-CSF-skull system by FEM and the present method (Eq. (23)). In the first 21 natural frequencies, one corresponds to flexural vibration $(n=1), 19$ to breathing vibration $(n \geq 2)$ and one to torsional vibration. 
Table 1. Coupled natural frequencies in the deformable skull case.

\begin{tabular}{rrccl}
\hline No. & $n$ & $\begin{array}{c}\text { Analytical frequency } \\
(\mathrm{rad} / \mathrm{s})\end{array}$ & $\begin{array}{c}\text { FEM frequency } \\
(\mathrm{rad} / \mathrm{s})\end{array}$ & Mode shape \\
\hline 1 & 1 & 0.043 & 0.043 & Flexural \\
2 & 0 & 0.049 & 0.049 & Torsional \\
3 & 13 & 0.1647 & 0.1647 & Breathing \\
4 & 14 & 0.1648 & 0.1648 & Breathing \\
5 & 12 & 0.1649 & 0.1649 & Breathing \\
6 & 15 & 0.1653 & 0.1653 & Breathing \\
7 & 11 & 0.1655 & 0.1655 & Breathing \\
8 & 16 & 0.1662 & 0.1662 & Breathing \\
9 & 10 & 0.1663 & 0.1663 & Breathing \\
10 & 9 & 0.1673 & 0.1673 & Breathing \\
11 & 17 & 0.1674 & 0.1674 & Breathing \\
12 & 8 & 0.1684 & 0.1684 & Breathing \\
13 & 18 & 0.1692 & 0.1692 & Breathing \\
14 & 7 & 0.1697 & 0.1697 & Breathing \\
15 & 6 & 0.1709 & 0.1709 & Breathing \\
16 & 19 & 0.1713 & 0.1713 & Breathing \\
17 & 5 & 0.1721 & 0.1721 & Breathing \\
18 & 4 & 0.1732 & 0.1732 & Breathing \\
19 & 20 & 0.1739 & 0.1739 & Breathing \\
20 & 3 & 0.1742 & 0.1742 & Breathing \\
21 & 2 & 0.1749 & 0.1749 & Breathing \\
\hline
\end{tabular}

Table 2. Coupled natural frequencies in the case of rigid skull.

\begin{tabular}{rrccl}
\hline No. & $n$ & $\begin{array}{c}\text { Analytical frequency } \\
(\mathrm{rad} / \mathrm{s})\end{array}$ & $\begin{array}{c}\text { FEM frequency } \\
\text { (rad/s) }\end{array}$ & Mode shape \\
\hline 1 & 1 & 0.043 & 0.043 & Flexural \\
2 & 0 & 0.049 & 0.049 & Torsional \\
3 & 13 & 0.1647 & 0.1647 & Breathing \\
4 & 14 & 0.1648 & 0.1648 & Breathing \\
5 & 12 & 0.1649 & 0.1649 & Breathing \\
6 & 15 & 0.1653 & 0.1653 & Breathing \\
7 & 11 & 0.1655 & 0.1655 & Breathing \\
8 & 16 & 0.1662 & 0.1662 & Breathing \\
9 & 10 & 0.1663 & 0.1663 & Breathing \\
10 & 9 & 0.1673 & 0.1673 & Breathing \\
11 & 17 & 0.1674 & 0.1674 & Breathing \\
12 & 8 & 0.1684 & 0.1684 & Breathing \\
13 & 18 & 0.1692 & 0.1692 & Breathing \\
14 & 7 & 0.1697 & 0.1697 & Breathing \\
15 & 6 & 0.1709 & 0.1709 & Breathing \\
16 & 19 & 0.1713 & 0.1713 & Breathing \\
17 & 5 & 0.1721 & 0.1721 & Breathing \\
18 & 4 & 0.1732 & 0.1732 & Breathing \\
19 & 20 & 0.1739 & 0.1739 & Breathing \\
20 & 3 & 0.1742 & 0.1742 & Breathing \\
21 & 2 & 0.1749 & 0.1749 & Breathing \\
\hline & & & &
\end{tabular}




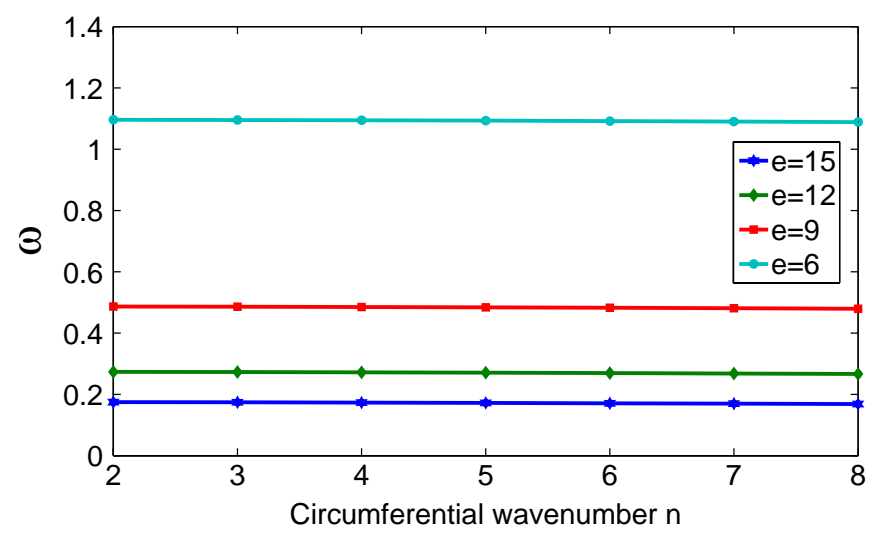

Fig. 2. Variations of the coupled breathing eigenmode for different CSF's thickness $e$ [mm] with the circumferential wavenumber $n$.

A very good agreement is observed between the results of the present method and those of FEM and the relative difference ((FEM-Present)/Present) is $\leq 1 \%$. This shows that the algorithm implemented in Comsol Multiphysics [Comsol, 2008] software for numerical computation is highly reliable and accurate. This algorithm is based on the UMFPACK method [Davis, 2003]. It is the attention to use the numerical formulation in future for more general geometries.

Tables 1 and 2 also show that the modal shapes are not in order with the parameters $n$. The frequency of mode $(n=15)$ is lower than that of mode $(n=2)$, or the frequency of mode $(n=16)$ is lower than that of mode $(n=3)$ for example. This feature of cylindrical vibration is different from that of beam vibration in which the order increases with the modal parameter. Therefore, in the vibration of the cylinder, one should be careful as to find the right mode of the vibration.

Six mode shapes are reported in Figs. 5 and 6 . The streamlines of components of velocity filed and the Von Mises stress field brain are represented since it plays a key role for understanding either the deep brain injury at low frequency loading. Mode shapes in Fig. 6 illustrate that the highest strain occurs at the interface when accounting for the fluid-interaction. It seems to suggest that traumatic brain injury at low frequency is localized at the interface.

In Fig. 6, we give some mode shapes of the brain. These mode shapes are not identical to those obtained in El Baroudi et al. [2009] where the inertial coupling is assumed. The fluid (CSF) domain becomes a diffusion layer. The localization phenomena of Von Mises stresses have completely disappeared and we obtain a regular distribution of Von Mises stresses.

In this paper, investigations are carried out to study the effects of CSF's thickness $e=R_{2}-R_{1}$ and elasticity of skull on the coupled natural frequencies BrainCSF-Skull system are presented with the present method.

First, one investigates how the coupled natural frequencies vary with the elasticity of skull $E_{2}$. When the skull is assumed to be elastic, we found the same 


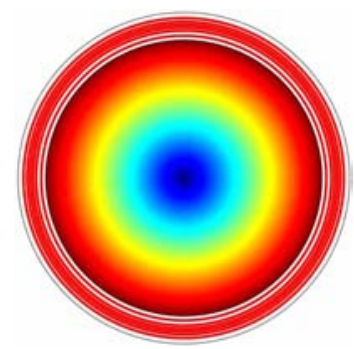

(a) $n=0$

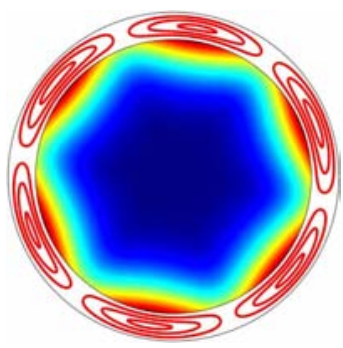

(d) $n=3$

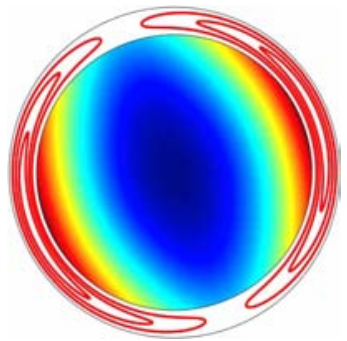

(b) $n=1$

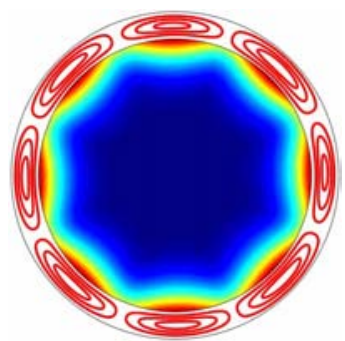

(e) $n=4$

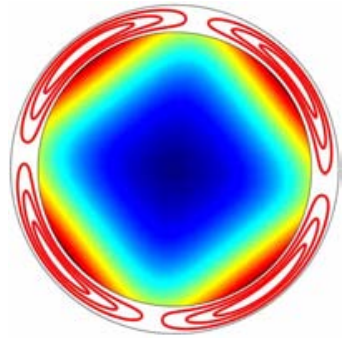

(c) $n=2$

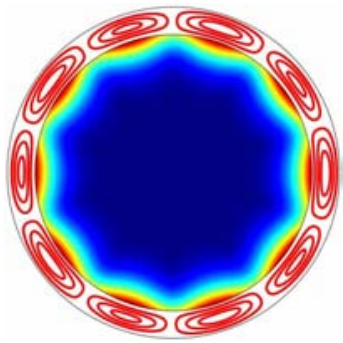

(f) $n=5$

Fig. 3. The coupled modal shapes of brain-CSF system in the case of rigid skull: the colors pertain to the displacement field of brain. Streamlines of components of velocity field of CSF.

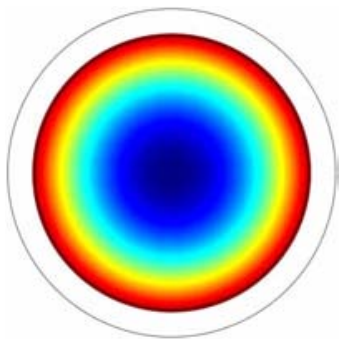

(a) $n=0$

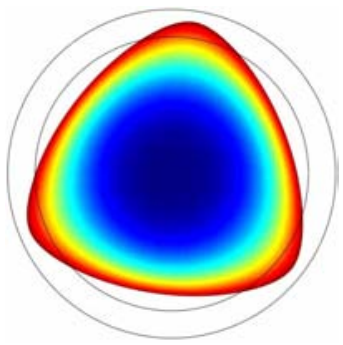

(d) $n=3$

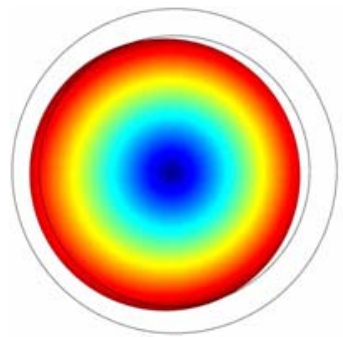

(b) $n=1$

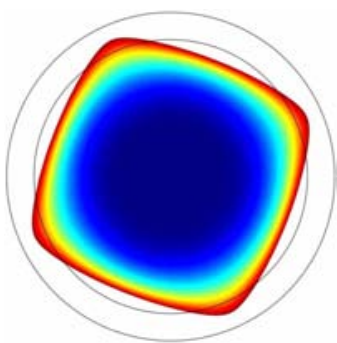

(e) $n=4$

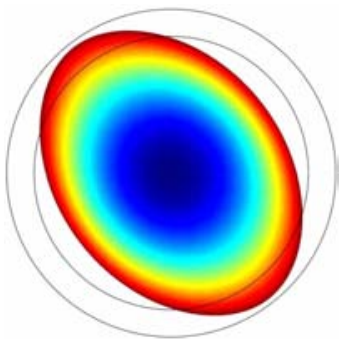

(c) $n=2$

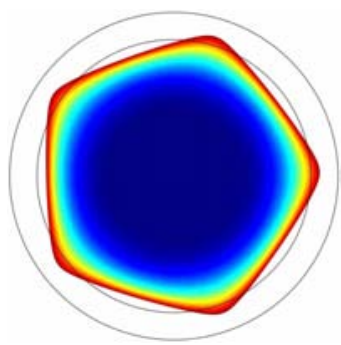

(f) $n=5$

Fig. 4. The modal shapes of Brain in the case of rigid skull: the colors pertain to the Von Mises stress. 


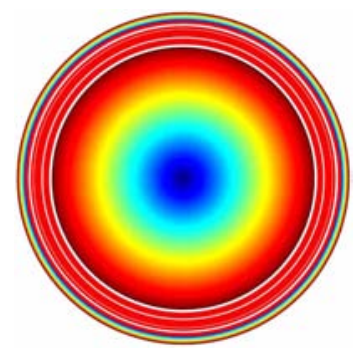

(a) $n=0$

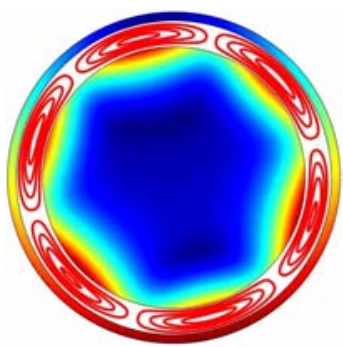

(d) $n=3$

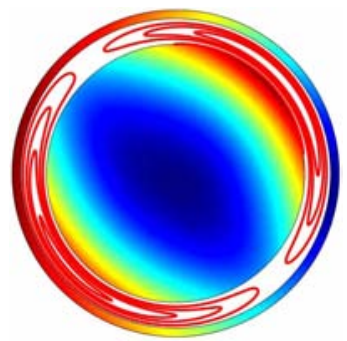

(b) $n=1$

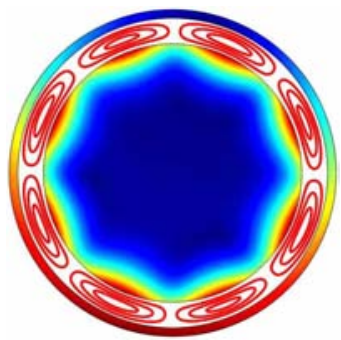

(e) $n=4$

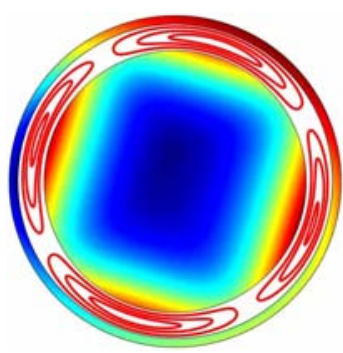

(c) $n=2$

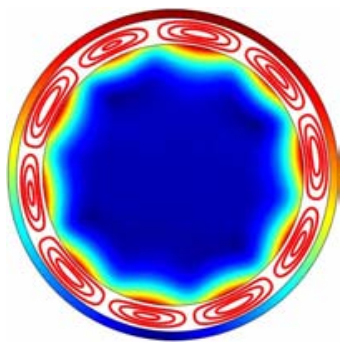

(f) $n=5$

Fig. 5. The coupled modal shapes of brain-CSF system in the deformable skull case: the colors pertain to the displacement field of brain. Streamlines of components of velocity field of CSF.

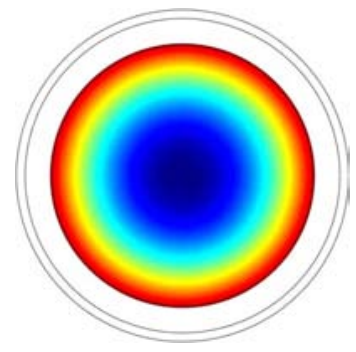

(a) $n=0$

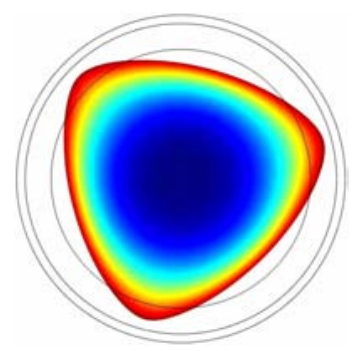

(d) $n=3$

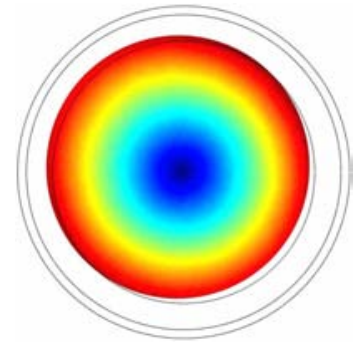

(b) $n=1$

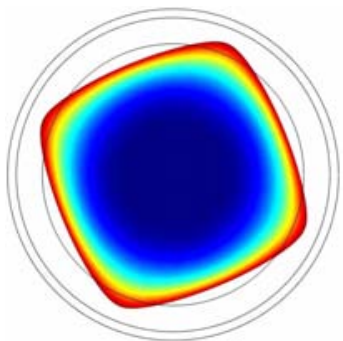

(e) $n=4$

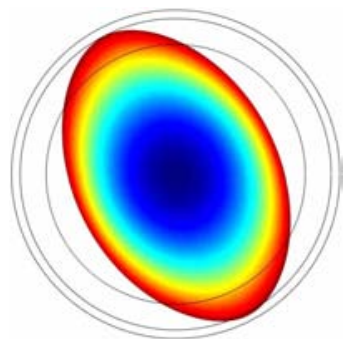

(c) $n=2$

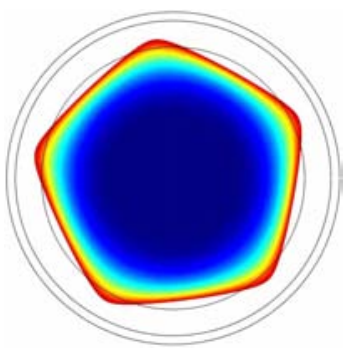

(f) $n=5$

Fig. 6. The modal shapes of brain in the deformable skull case: the colors pertain to the Von Mises stress. 
natural frequencies and mode shapes as for the rigid skull (Tables 1 and 2 and Figs. 3-6). The natural frequencies do not vary significantly even for a large band $E_{2}>4.7[\mathrm{GPa}]$ which is the Youngs modulus of a childs skull of three years old [El Baroudi et al., 2012a].

Secondly, investigations are carried out to study the effects of CSF characteristics such as thickness on the coupled natural frequencies of brain-CSF-skull system. To this purpose, we take for the CSF radius $R_{2}$ and we vary $e$. Figure 2 shows that the CSF thickness has a strong influence on the natural frequencies. The natural frequencies increases when the CSF thickness decreases due to added mass [Ohayon and Soize, 1997].

\section{Conclusion}

This paper investigates coupled natural frequencies of an idealized human head. The solutions satisfy all the governing equations and boundary conditions point by point and they are two-dimensional exact. The numerical example shows a good agreement between the results of the present method and those of FEM. The knowledge of the natural frequencies may be very important either in understanding Trauma Brain Injury during impacted head [Willinger et al., 1995]; or neuroimaging [Will and Berg, 2007] and [Hiltunen et al., 2006]. Very simplified models have been developed in the present study for analyzing the influence of the CSF phase on the frequency spectrum on the brain-CSF-skull system. Some concluding remarks could be drawn:

- The analytical and numerical results are in good agreement in this study. It is the attention to use it in the future for more general geometries.

- The CSF thickness has a strong influence on the natural frequencies. Indeed, decreasing the fluid thickness tends to increase the frequencies since the added mass increases accordingly.

- Mode shapes of the rigid skull are the same as those of the elastic skull case. This is probably due to the high stiffness of the skull compared to that of the brain. In such a case, it is therefore possible to replace the "stiff skull" by a infinitely rigid wall. But in all cases, it seems not a very realistic model for analyzing the head impact.

- The model El Baroudi et al. [2009] when the inertial coupling is assumed and the present study do not give similar results. This means that the results depend strongly on the choice of coupling used.

\section{Acknowledgments}

We would like to sincerely thank Professor L. Rakotomanana for his useful comments and suggestions. 


\section{Appendix A}

The matrix $\mathbf{M}$ in Eq. (22) in the deformable skull case is defined as follows:

$$
\mathbf{M}=\left[\begin{array}{cccccccccc}
b_{11} & b_{12} & b_{13} & b_{14} & b_{15} & b_{16} & 0 & 0 & 0 & 0 \\
b_{21} & b_{22} & b_{23} & b_{24} & b_{25} & b_{26} & 0 & 0 & 0 & 0 \\
b_{31} & b_{32} & b_{33} & b_{34} & b_{35} & b_{36} & 0 & 0 & 0 & 0 \\
b_{41} & b_{42} & b_{43} & b_{44} & b_{45} & b_{46} & 0 & 0 & 0 & 0 \\
0 & 0 & b_{53} & b_{54} & b_{55} & b_{56} & b_{57} & b_{58} & b_{59} & b_{510} \\
0 & 0 & b_{63} & b_{64} & b_{65} & b_{66} & b_{67} & b_{68} & b_{69} & b_{610} \\
0 & 0 & b_{73} & b_{74} & b_{75} & b_{76} & b_{77} & b_{78} & b_{79} & b_{710} \\
0 & 0 & b_{83} & b_{84} & b_{85} & b_{86} & b_{87} & b_{88} & b_{89} & b_{810} \\
0 & 0 & 0 & 0 & 0 & 0 & b_{97} & b_{98} & b_{99} & b_{1010} \\
0 & 0 & 0 & 0 & 0 & 0 & b_{107} & b_{108} & b_{109} & b_{1010}
\end{array}\right],
$$

where:

$$
\begin{aligned}
& b_{11}=\nu k_{\psi}^{2} \frac{d \Phi_{1}^{(1)}}{d r}\left(R_{1}\right) \\
& b_{12}=-\frac{\nu k_{\psi}^{2} n}{R_{1}} \Psi_{1}^{(1)}\left(R_{1}\right) \\
& b_{13}=\frac{d \phi_{1}}{d r}\left(R_{1}\right) \\
& b_{14}=\frac{d \phi_{2}}{d r}\left(R_{1}\right) \\
& b_{15}=-\frac{n}{R_{1}} \psi_{1}\left(R_{1}\right) \\
& b_{16}=-\frac{n}{R_{1}} \psi_{2}\left(R_{1}\right) \\
& b_{21}=\frac{\nu k_{\psi}^{2} n}{R_{1}} \Phi_{1}^{(1)}\left(R_{1}\right) \\
& b_{22}=-\nu k_{\psi}^{2} \frac{d \Psi_{1}^{(1)}}{d r}\left(R_{1}\right) \\
& b_{23}=\frac{n}{R_{1}} \phi_{1}\left(R_{1}\right) \\
& b_{24}=\frac{n}{R_{1}} \phi_{2}\left(R_{1}\right) \\
& b_{25}=-\frac{d \psi_{1}}{d r}\left(R_{1}\right) \\
& b_{1}
\end{aligned}
$$




$$
\begin{aligned}
& b_{26}=-\frac{d \psi_{2}}{d r}\left(R_{1}\right) \\
& b_{31}=\frac{d^{2} \Phi_{1}^{(1)}}{d r^{2}}\left(R_{1}\right)+\lambda_{1} k_{L_{1}}^{2} \Phi_{1}^{(1)}\left(R_{1}\right) \\
& b_{32}=-\frac{2 \mu_{1} n}{R_{1}^{2}}\left[R_{1} \frac{d \Psi_{1}^{(1)}}{d r}\left(R_{1}\right)-\Psi_{1}^{(1)}\left(R_{1}\right)\right] \\
& b_{33}=\eta\left[2 \frac{d^{2} \phi_{1}}{d r^{2}}\left(R_{1}\right)-k_{\psi}^{2} \phi_{1}\left(R_{1}\right)\right] \\
& b_{34}=\eta\left[2 \frac{d^{2} \phi_{2}}{d r^{2}}\left(R_{1}\right)-k_{\psi}^{2} \phi_{2}\left(R_{1}\right)\right] \\
& b_{35}=\frac{2 n \eta}{R_{1}^{2}}\left[\psi_{1}\left(R_{1}\right)-R_{1} \frac{d \psi_{1}}{d r}\left(R_{1}\right)\right] \\
& b_{36}=\frac{2 n \eta}{R_{1}^{2}}\left[\psi_{2}\left(R_{1}\right)-R_{1} \frac{d \psi_{2}}{d r}\left(R_{1}\right)\right] \\
& b_{41}=\frac{2 \mu_{1} n}{R_{1}^{2}}\left[R_{1} \frac{d \Phi_{1}^{(1)}}{d r}\left(R_{1}\right)-\Phi_{1}^{(1)}\left(R_{1}\right)\right] \\
& b_{42}=-2 \mu_{1}\left[\frac{d^{2} \Psi_{1}^{(1)}}{d r^{2}}\left(R_{1}\right)-\frac{k_{T_{1}}^{2}}{2} \Psi_{1}^{(1)}\left(R_{1}\right)\right] \\
& b_{43}=\frac{2 n \eta}{R_{1}^{2}}\left[R_{1} \frac{d \phi_{1}}{d r}\left(R_{1}\right)-\phi_{1}\left(R_{1}\right)\right] \\
& b_{44}=\frac{2 n \eta}{R_{1}^{2}}\left[R_{1} \frac{d \phi_{2}}{d r}\left(R_{1}\right)-\phi_{2}\left(R_{1}\right)\right] \\
& b_{45}=-\eta\left[2 \frac{d^{2} \psi_{1}}{d r^{2}}\left(R_{1}\right)+k_{\psi}^{2} \psi_{1}\left(R_{1}\right)\right] \\
& b_{46}=-\eta\left[2 \frac{d^{2} \psi_{2}}{d r^{2}}\left(R_{1}\right)+k_{\psi}^{2} \psi_{2}\left(R_{1}\right)\right] \\
& b_{53}=\frac{d \phi_{1}}{d r}\left(R_{2}\right) \\
& b_{54}=\frac{d \phi_{2}}{d r}\left(R_{2}\right) \\
& b_{55}=-\frac{n}{R_{2}} \psi_{1}\left(R_{2}\right) \\
& b_{56}=-\frac{n}{R_{2}} \psi_{2}\left(R_{2}\right)
\end{aligned}
$$




$$
\begin{aligned}
b_{57} & =\nu k_{\psi}^{2} \frac{d \Phi_{1}^{(2)}}{d r}\left(R_{2}\right) \\
b_{58} & =\nu k_{\psi}^{2} \frac{d \Phi_{2}^{(2)}}{d r}\left(R_{2}\right) \\
b_{59} & =-\frac{\nu k_{\psi}^{2} n}{R_{2}} \Psi_{1}^{(2)}\left(R_{2}\right) \\
b_{510} & =-\frac{\nu k_{\psi}^{2} n}{R_{2}} \Psi_{2}^{(2)}\left(R_{2}\right) \\
b_{63} & =\frac{n}{R_{2}} \phi_{1}\left(R_{2}\right) \\
b_{64} & =\frac{n}{R_{2}} \phi_{2}\left(R_{2}\right) \\
b_{65} & =-\frac{d \psi_{1}}{d r}\left(R_{2}\right) \\
b_{77} & =2 \mu_{2}\left[\frac{d^{2} \Phi_{1}^{(2)}}{d r^{2}}\left(R_{2}\right)+\frac{\lambda_{2} k_{L_{2}}^{2}}{2 \mu_{2}} \Phi_{1}^{(2)}\left(R_{2}\right)\right] \\
b_{66} & =-\frac{d \psi_{2}}{d r}\left(R_{2}\right) \\
b_{75} & =-\frac{2 n \eta}{R_{2}^{2}}\left[R_{2} \frac{d \psi_{1}}{d r}\left(R_{2}\right)-\psi_{1}\left(R_{2}\right)\right] \\
b_{74} & =\eta\left[2 \frac{d^{2} \phi_{2}}{d r^{2}}\left(R_{2}\right)-k_{\psi}^{2} \phi_{2}\left(R_{2}\right)\right] \\
b_{67} & =\frac{\nu k_{\psi}^{2} n}{R_{1}} \Phi_{1}^{(2)}\left(R_{2}\right) \\
b_{69} & =-\nu k_{\psi}^{2} \frac{d \Psi_{1}^{2}}{d r}\left(R_{2}^{2} n \Psi_{2}^{(2)}\left(R_{2}\right)\right. \\
b_{68} & \left.=\frac{d^{2} \phi_{1}}{d r_{1}^{2}}\left(R_{2}\right)-k_{2}^{2} \phi_{1}\left(R_{2}\right)\right]
\end{aligned}
$$




$$
\begin{aligned}
& b_{78}=2 \mu_{2}\left[\frac{d^{2} \Phi_{2}^{(2)}}{d r^{2}}\left(R_{2}\right)+\frac{\lambda_{2} k_{L_{2}}^{2}}{2 \mu_{2}} \Phi_{2}^{(2)}\left(R_{2}\right)\right] \\
& b_{79}=-\frac{2 \mu_{2} n}{R_{2}^{2}}\left[R_{2} \frac{d \Psi_{1}^{(2)}}{d r}\left(R_{2}\right)-\Psi_{1}^{(2)}\left(R_{2}\right)\right] \\
& b_{710}=-\frac{2 \mu_{2} n}{R_{2}^{2}}\left[R_{2} \frac{d \Psi_{2}^{(2)}}{d r}\left(R_{2}\right)-\Psi_{2}^{(2)}\left(R_{2}\right)\right] \\
& b_{83}=\frac{2 n \eta}{R_{2}^{2}}\left[R_{2} \frac{d \phi_{1}}{d r}\left(R_{2}\right)-\phi_{1}\left(R_{2}\right)\right] \\
& b_{84}=\frac{2 n \eta}{R_{2}^{2}}\left[R_{2} \frac{d \phi_{2}}{d r}\left(R_{2}\right)-\phi_{2}\left(R_{2}\right)\right] \\
& b_{85}=-\eta\left[2 \frac{d^{2} \psi_{1}}{d r^{2}}\left(R_{2}\right)+k_{\psi}^{2} \psi_{1}\left(R_{2}\right)\right] \\
& b_{86}=-\eta\left[2 \frac{d^{2} \psi_{2}}{d r^{2}}\left(R_{2}\right)+k_{\psi}^{2} \psi_{2}\left(R_{2}\right)\right] \\
& b_{87}=\frac{2 \mu_{2} n}{R_{2}^{2}}\left[R_{2} \frac{d \Phi_{1}^{(2)}}{d r}\left(R_{2}\right)-\Phi_{1}^{(2)}\left(R_{2}\right)\right] \\
& b_{88}=\frac{2 \mu_{2} n}{R_{2}^{2}}\left[R_{2} \frac{d \Phi_{2}^{(2)}}{d r}\left(R_{2}\right)-\Phi_{2}^{(2)}\left(R_{2}\right)\right] \\
& b_{89}=-2 \mu_{2}\left[\frac{d^{2} \Psi_{1}^{(2)}}{d r^{2}}\left(R_{2}\right)-\frac{k_{T_{2}}^{2}}{2} \Psi_{1}^{(2)}\left(R_{2}\right)\right] \\
& b_{810}=-2 \mu_{2}\left[\frac{d^{2} \Psi_{2}^{(2)}}{d r^{2}}\left(R_{2}\right)-\frac{k_{T_{2}}^{2}}{2} \Psi_{2}^{(2)}\left(R_{2}\right)\right] \\
& b_{97}=2 \mu_{2}\left[\frac{d^{2} \Phi_{1}^{(2)}}{d r^{2}}\left(R_{0}\right)+\frac{\lambda_{2} k_{L_{2}}^{2}}{2 \mu_{2}} \Phi_{1}^{(2)}\left(R_{0}\right)\right] \\
& b_{98}=2 \mu_{2}\left[\frac{d^{2} \Phi_{2}^{(2)}}{d r^{2}}\left(R_{0}\right)+\frac{\lambda_{2} k_{L_{2}}^{2}}{2 \mu_{2}} \Phi_{2}^{(2)}\left(R_{0}\right)\right] \\
& b_{99}=-\frac{2 \mu_{2} n}{R_{0}^{2}}\left[R_{0} \frac{d \Psi_{1}^{(2)}}{d r}\left(R_{0}\right)-\Psi_{1}^{(2)}\left(R_{0}\right)\right] \\
& b_{910}=-\frac{2 \mu_{2} n}{R_{0}^{2}}\left[R_{0} \frac{d \Psi_{2}^{(2)}}{d r}\left(R_{0}\right)-\Psi_{2}^{(2)}\left(R_{0}\right)\right]
\end{aligned}
$$




$$
\begin{aligned}
& b_{107}=\frac{2 \mu_{2} n}{R_{0}^{2}}\left[R_{0} \frac{d \Phi_{1}^{(2)}}{d r}\left(R_{0}\right)-\Phi_{1}^{(2)}\left(R_{0}\right)\right] \\
& b_{108}=\frac{2 \mu_{2} n}{R_{0}^{2}}\left[R_{0} \frac{d \Phi_{2}^{(2)}}{d r}\left(R_{0}\right)-\Phi_{2}^{(2)}\left(R_{0}\right)\right] \\
& b_{109}=-2 \mu_{2}\left[\frac{d^{2} \Psi_{1}^{(2)}}{d r^{2}}\left(R_{0}\right)-\frac{k_{T_{2}}^{2}}{2} \Psi_{1}^{(2)}\left(R_{0}\right)\right] \\
& b_{1010}=-2 \mu_{2}\left[\frac{d^{2} \Psi_{2}^{(2)}}{d r^{2}}\left(R_{0}\right)-\frac{k_{T_{2}}^{2}}{2} \Psi_{2}^{(2)}\left(R_{0}\right)\right] .
\end{aligned}
$$

In the case, when the skull is assumed to be rigid, the matrix $\mathbf{M}$ becomes:

$$
\mathbf{M}=\left[\begin{array}{cccccc}
b_{11} & b_{12} & b_{13} & b_{14} & b_{15} & b_{16} \\
b_{21} & b_{22} & b_{23} & b_{24} & b_{25} & b_{26} \\
b_{31} & b_{32} & b_{33} & b_{34} & b_{35} & b_{36} \\
b_{41} & b_{42} & b_{43} & b_{44} & b_{45} & b_{46} \\
0 & 0 & b_{53} & b_{54} & b_{55} & b_{56} \\
0 & 0 & b_{63} & b_{64} & b_{65} & b_{66}
\end{array}\right] .
$$

\section{References}

Axisa, F. and Antunes, J. [2007] Modelling of Mechanical Systems: Fluid-Structure Interaction, Vol. 3 (Elsevier, Oxford).

Charalambopoulos, A., Dassios, G., Fotiadis, D. and Massalas, C. [2006] "Frequency spectrum of the human head-neck system," International Journal of Engineering Science 35, $753-768$.

Chen, H. and Luo, Y. [2010] "Parametric study of closed head injuries," Advances in Theoretical and Applied Mechanics 3, 339-347.

Comsol Multiphysics [2008] Comsol Multiphysics 3.5a: Reference and User's Guide.

Davis, T. A. [2003] "Algorithm 832: UMFPACK, an unsymmetric-pattern multifrontal method," ACM Transactions on Mathematical Software 34(2), 165-199.

El Baroudi, A., Razafimahery, F. and Rakotomanana, L. [2009] "Parametric modal analysis of brain-CSF-skull system: Influence of fluid-structure interaction," European Journal of Computational Mechanics 18(1), 55-66.

El Baroudi, A., Razafimahery, F. and Rakotomanana, L. [2012a] "Three-dimensional modal analysis of an idealized human head including fluid-structure interaction effects," Acta Mechanica 223, 1899-1915.

El Baroudi, A., Razafimahery, F. and Rakotomanana, L. [2012b] "Study of a spherical head model. Analytical and numerical solutions in fluid-structure interaction approach," International Journal of Engineering Science 51, 1-13.

Hiltunen, J., Hari, R., Jousmaki, V. and Mueller, K. [2006] "Quantification of mechanical vibration during diffusion tensor imaging at 3 T," NeuroImage 32, 93-103.

Kleiven, S. [2002] Finite element modeling of the human head, Doctoral Thesis, Technical Report 2002-9, Royal Institute of Technology. 
Liu, Z. S., Luo, X. Y., Lee, H. P. and Lu, C. [2007] "Snoring source identification and snoring noise prediction," Journal of Biomechanics 40, 861-870.

Marjoux, D., Baumgartner, D., Deck, C. and Willinger, R. [2008] "Head injury prediction capability of the HIC, HIP, SIMon and ULP criteria," Accident Analysis and Prevention 40, 1135-1148.

Morand, H. J. P. and Ohayon, R. [1979] "Substructure variational analysis of the vibrations of coupled fluid-structure systems. Finite element results," International Journal of Numerical Methods and Engineering 14, 741-755.

Morse, M. and Feshbach, H. R. [1946] Methods of Theoretical Physics (McGraw-Hill, New York).

Ohayon, R. and Soize, C. [1997] Structural Acoustics and Vibration (Academic Press, London).

Paidoussis, M. P. [1997] Fluid-Structure Interactions Slender Structures and Axial Flow, Vol. 1 (Academic Press, London).

Verschuerena, P., Delyeb, H., Depreitere, B., Van Lierde, C., Haex, B., Berckmans, D., Verpoestd, I., Goffin, J., Vander, J. and Van der Perre, G. [2007] "A new test set-up for skull fracture characterisation," Journal of Biomechanics 40, 3389-3396.

Will, U. and Berg, E. [2007] "Brain wave synchronization and entrainment to periodic acoustic stimuli," Neuroscience Letters 424, 55-60.

Willinger, R., Taleb, L. and Kopp, C. M. [1995] "Modal and temporal analysis of head mathematical models," Journal of Neurotrauma 12(4), 743-754.

Zong, Z., Lee, H. P. and Lu, C. [2006] "A three-dimensional human head finite element model and power flow in a human head subject to impact loading," Journal of Biomechanics 39, 284-292. 